\title{
ON THE SPACE OF RIEMANNIAN METRICS ${ }^{1}$
}

BY DAVID G. EBIN ${ }^{2}$

Communicated by S. Smale, March 20, 1968

1. Results. In the present announcement we are concerned with the space of Riemannian metrics on a compact smooth manifold. Let $M$ be such a manifold, $S^{2} T^{*}$ the bundle of symmetric covariant twotensors on $M$, and $C^{\infty}\left(S^{2} T^{*}\right)$ the smooth sections of this bundle, endowed with the $C^{\infty}$ topology. If $\mathfrak{N} \subseteq C^{\infty}\left(S^{2} T^{*}\right)$ is the set of smooth Riemannian metrics on $M$ (those sections which at each point $p$ of $M$ induce a positive definite bilinear form on $T_{p}$, the tangent space to $M$ ), it is well known that $\mathscr{T}$ is an open convex cone in $C^{\infty}\left(S^{2} T^{*}\right)$. If $D$ is the group of diffeomorphisms of $M$ (with the $C^{\infty}$ topology), $D$ acts on $C^{\infty}\left(S^{2} T^{*}\right)$ on the right by "pull-back" and $\mathfrak{T}$ is an invariant set under the action. We write $A: D \times C^{\infty}\left(S^{2} T^{*}\right) \rightarrow C^{\infty}\left(S^{2} T^{*}\right)$ and denote $A(\eta, \gamma)$ by $\eta^{*}(\gamma) . A$ is a right action because $(\xi \eta)^{*} \gamma=\eta^{*} \xi^{*}(\gamma)$.

Now restrict to $A: D \times \mathfrak{M} \rightarrow \mathfrak{N}$. For any $\lambda \in \mathscr{N}$ define $I_{\lambda}$, the isotropy group of $\lambda$, by $I_{\lambda}=\left\{\eta \in D \mid \eta^{*}(\lambda)=\lambda\right\}$. For a fixed $\gamma \in \Re$, let $O_{\gamma}$ be the orbit of $D$ through $\gamma$.

MAIN Theorem.

(1) $A$ induces a homeomorphism of $D / I_{\gamma}$ onto $O_{\gamma}$ by $\eta I_{\gamma} \rightarrow \eta^{*}(\gamma)$.

(2) There is a subspace $S$ of $\mathbb{N}$ containing $\gamma$ which has the following properties:

(a) $A\left(I_{\gamma}, S\right)=S$,

(b) If $\eta \in \mathscr{D}$ and $\eta^{*}(S) \cap S \neq \varnothing$, then $\eta \in I_{\gamma}$,

(c) There exists a neighborhood $U$ of the identity coset in $D / I_{\gamma}$ and a local cross section $\chi: U \rightarrow D$ such that the map $F: U \times S \rightarrow \mathfrak{N}$ by $F(u, s)=(\chi(u))^{*}(s)$ is a homeomorphism onto a neighborhood of $\gamma$.

$S$ is called a slice through $\gamma$. From this theorem it follows easily that for any $\lambda \in \Re$ which is sufficiently near $\gamma$, there exists $\eta \in D$ such that $I_{\lambda} \subseteq \eta I_{\gamma} \eta^{-1}$.

$I_{\gamma}$ is by definition the group of isometrics of $\Re$ with respect to the metric $\gamma$, so we have shown that this group cannot increase locally. In particular we know that the set $\mathcal{G}$ of metrics which have trivial [2].

${ }^{1}$ Most of the results presented here appeared in the author's doctoral dissertation

2 Partially supported by N.S.F. post-doctoral fellowship. 
isometry group is open in $\Re$. If $\operatorname{dim} M=1, \oint=\varnothing$. If $\operatorname{dim} M>1$, we can show that $\mathcal{G}$ is dense in $\mathfrak{T}$. Therefore we call it the set of generic metrics.

2. Outline of proofs. The main theorem as well as its proof is inspired by the case of an action of a compact Lie group. If $D$ is replaced by such a group, $\mathfrak{T}$ replaced by a differentiable manifold and the action $A$ is differentiable, then our theorem is a well-known result (see $[1$, p. 108]). To prove it, one constructs a D-invariant Riemannian metric on $\Re$, looks at the normal bundle of $\nu$ of $O_{\gamma}$ and defines $S$ as the image under the exponential map of a small ball about the origin in the fibre of $\nu$ above $\gamma$.

In the case at hand, it is not feasible to give $\mathfrak{T}$ a manifold structure because $\mathfrak{T}$ is locally like a Fréchet space. Therefore we enlarge it to the space $\Re \tau^{s}$, the set of sections of $S^{2} T^{*}$ which induce positive definite forms at each point of $M$ and which belong to the Sobolev space $H^{s}\left(S^{2} T^{*}\right)$. (See [3], or [4] where this is called $L_{k}^{2}\left(S^{2} T^{*}\right)$.) Also we enlarge $\mathscr{D}$ to $D^{s+1}$, the set of $H^{s+1}$ maps of $M$ to $M$ which have $H^{s+1}$ inverses (see [4] where they are constructed as sections of fibre bundles.) $D^{s+1}$ turns out to be a group, $\mathfrak{D}^{s+1}$ and $\mathfrak{M}^{s}$ are Hilbert manifolds, and $A$ extends to a continuous action $A: D^{8+1} \times \mathfrak{N} \mathbb{1}^{8} \rightarrow \mathbb{N}^{8}$. Also, if $\gamma \in \mathscr{N}, \psi_{\gamma}=A\left\lceil D^{s+1} \times\{\gamma\} \rightarrow \mathbb{N}^{\circ}\right.$ is smooth, and its image $O_{\gamma}^{s}$ is a submanifold of $\Re^{s} .^{3}$

Using covariant derivatives and jet bundles on $M$ (as in [3, p. 90]) we construct a natural Riemannian metric on $\mathfrak{T l}^{8}$, which is $D^{8+1}$ invariant. Then using the normal bundle $\nu^{8}$ to $O_{\gamma}^{s}$ and the exponential map $\exp _{s}$ on $\mathfrak{T}^{s}$, we get (2) of the main theorem except that in (b) we must restrict to the case where $\eta$ is close to the identity.

Since $\exp _{s}$ commutes with the action of $D$, it maps the smooth elements of $\nu_{s}$ into $\mathscr{T}$ and is a local homeomorphism with respect to the $C^{\infty}$ topologies as well. Using the fact that if a sequence of Riemannian metrics on $M$ converges $C^{\infty}$, then so does the sequence of exponential maps associated to them, we prove (1) of the theorem for the smooth case. This together with the above implies all of (2) as well.

To prove that $G \subseteq \mathscr{T}$ is dense in the case $\operatorname{dim} M>1$, we look at the curvature of $M$. By perturbing the metric $\gamma$ to some $\gamma^{\prime}$ close to it, we can cause the curvature to be such that $I_{\gamma^{\prime}}$ must be very near the

${ }^{3}$ Here we need to know that the tangent space to $O_{\gamma}^{*}$ is everywhere a closed subspace of the tangent space to T/s. This follows from the fact that $T \psi_{\gamma}$, the map induced by $\psi_{\gamma}$ on the tangent bundles, looks like a differential operator with injective symbol. 
identity of D. But $I_{\gamma^{\prime}} \subseteq \eta I_{\gamma} \eta^{-1}$ for $\eta$ near the identity, so this is impossible since it would mean that $I_{\gamma}$ would have small subgroups.

The author wishes to thank Professor I. M. Singer for his many fruitful ideas.

\section{BIBLIOGRAPHY}

1. A. Borel, Seminar on transformation groups, Ann. of Math. Studies No. 46, Princeton Univ. Press, Princeton, N. J., 1960.

2. D. G. Ebin, On the space of Riemannian metrics, Doctoral Thesis, Massachusetts Institute of Technology, Cambridge, Mass., 1967.

3. R. S. Palais, Seminar on the Atiyah-Singer index theorem, Ann. of Math. Studies No. 57, Princeton Univ. Press, Princeton, N. J., 1965.

4. - Foundations of global non-linear analysis, W. A. Benjamin, New York, 1968.

University of California, Berkeley 